\title{
Cinética de degradación ruminal in situ de ensilado de rastrojo de maíz (Zea mays) con niveles crecientes de urea
}

\author{
Sánchez, A. '; Perea, J. ${ }^{2}$; Montenegro, L. '; Espinoza, I. ${ }^{\text {; }}$ Avellaneda, J. ${ }^{\text {' }}$ and Barba, C. ${ }^{2 @}$
}

'Facutad de Ciencias Pecuarias. Universidad Técnica Estatal de Quevedo. Quevedo, Los Ríos. Ecuador.

2Departamento de Producción Animal. Universidad de Córdoba. España.

PALABRAS CLAVE

Subproductos agroindustriales.

Conservación de forrajes.

Sostenibilidad.

Ganado criollo.

Economía circular.

\section{RESUMEN}

Se estudió el efecto de la inclusión de urea sobre la composición y cinética de degradación ruminal in situ en el ensilado de rastrojo de maíz. Se partió de cuatro de tratamientos al adicionar el 0, 1, 2 y $3 \%$ de urea a una mezcla de rastrojo de maíz con el $10 \%$ de melaza. Se elaboraron 128 microsilos 3 $\mathrm{kg} /$ unidad y 4 repeticiones por tratamiento). La apertura de los silos se realizó a los 7, 14, 21, 28, 35, 42,49 y 56 días. Se determinó el contenido de la materia seca (MS), materia orgánica (MO), proteína bruta (PB), FND y FAD. La degradabilidad in situ se obtuvo mediante el método de Mehrez y Orskov y la degradabilidad de MS y PB mediante el análisis de los residuos con el método Kieldhal. Los datos se analizaron con el procedimiento GLM y el test de Tukey $\left(\mathrm{P}_{ \pm<<0.05)}\right)$ del SAS, la opción CONTRAST y la función SOLVER de Microsoff EXCEL®. El mejor comportamiento se observó a los 35 días de ensilado $(p<0,05)$. Se evidenció la existencia de diferencias significativas para todas las variables excepto $M O$. La adición de urea se relacionó con el incremento de MS y PB y con el descenso de valores de FND y FAD ( $p<0,01)$. La degradabilidad ruminal In Situ de MS ascendió conforme aumentó el nivel de urea, excepto en el caso del factor $\mathrm{C}$, mientras que para PB no reflejó diferencias entre los distintos tratamientos. La degradabilidad efectiva fue superior en MS, y con mayor variabilidad entre tratamientos, que en PB donde se apreció un comportamiento más homogéneo. La adición de urea al 3\% mejora la composición nutricional y degradabilidad del ensilado de rastrojo de maíz con melaza, lo que hace posible que los pequeños productores dispongan de una alternativa de suplementación animal eficiente y aceptable ambientalmente con esta técnica.

\section{In situ degradation kinetic of maize stubble silage (Zea mays) with increasing levels of urea}

\section{SUMMARY}

The urea inclusion effect on the nutritional composition and kinetics of in situ ruminal digestion on maize stubble silage was studied. Four treatments were started by adding $0,1,2$ and $3 \%$ urea to a mixture of maize stubble with $10 \%$ molasses. 128 microsilos ( $3 \mathrm{~kg} /$ unit and 4 repetitions per treatment) were made. Experimental silages were opened at $7,14,21,28,35,42,49$ and 56 days. The content of dry matter $(M S)$, organic matter $(O M)$, crude protein (PB), FND and FAD were determined. In situ degradability was obtained by Mehrez and Orskov method and the degradability of MS and PB by residue analysis with Kjeldhal method. A general linear model was used for data manipulation, using the statistical package SAS (2004), as well as Contrast option and Solver function of Microsoft EXCEL $囚$. 35-days conservation silage $(p<0.05)$ was observed as best treatment. Significant differences for all the variables except $O M$ was evidenced. Urea addition was related to increase in $M S$ and $P B$ and decrease in FND and FAD values $P$ $<0.01$ ). In situ ruminal degradability of DM increased as urea level increased, except in the case of factor $\mathrm{c}$, while for PB differences between treatments were not reflected. Urea addition was related to increase in $M S$ and $P B$ and decrease in FND and FAD values $(p<0.01)$. In situ ruminal degradability of $D M$ increased as urea level increased, except in case of factor $c$, while differences between treatments for PB were not reflected. Effective degradability was higher in $M S$, and with greater variability between treatments than in $\mathrm{PB}$, where a more homogeneous behavior was observed. Addition of $3 \%$ urea improves the nutritional composition and degradability of maize stubble silage with molasses, making it possible for smallhorders to have an efficient and environmentally acceptable alternative animal feed supplement with this technique..

\section{INTRODUCCIÓN}

La cría de ganado bovino criollo de doble propósito es la actividad pecuaria predominante en las regiones tropicales de Latinoamérica (Torres et al., 2015). La alimentación del ganado de doble propósito se basa fundamentalmente en la utilización de pastos naturales y cultivados de no muy alta calidad, así como en el em- pleo de cantidades crecientes de residuos de cultivos y subproductos agroindustriales (Montenegro et al., 2018). En el caso de los pastos, existe una clara estacionalidad en la producción de biomasa con grandes diferencias entre la época lluviosa ( $80 \%$ del total) frente a la menor productividad de la época seca (Caraballo et al., 2007), por cuanto se compromete el desarrollo de la 
producción ganadera y la mejora sostenida del sector agropecuario en estas latitudes. De acuerdo con Rangel et al. (2020) hay carencia de programas de manejo y mejora de la producción de forraje verde y, en el caso de los cultivos, la producción de granos tiene prioridad para el consumo humano, con independencia de que parte de los mismos se estén desviando hacia la producción de biocombustibles.

La utilización de residuos de cosecha y subproductos agroindustriales en alimentación animal resulta estratégica para alcanzar un desarrollo territorial y favorecer la soberanía alimentaria de modo sostenible (García et al., 2016). Además, el empleo de los subproductos permite aumentar su ciclo de vida y reutilización, además de contribuir a mitigar sus efectos en el medio ambiente (Pardo et al., 2016). Sin embargo, el uso de la mayor parte de estos residuos es ineficaz por su escasa estabilidad y durabilidad en condiciones tropicales, entre otros limitantes y desventajas, así como la falta de conocimiento sobre su valoración, estrategias de conservación, pautas de uso e implementación en dietas (Meneses et al., 2007).

El ensilaje puede constituir una técnica adecuada de conservación apropiada para los subproductos en el trópico dado su alto contenido acuoso (Cándido et al. 2007), así como por depender en menor medida de las condiciones climáticas en comparación con la henificación (Sánchez-Laiño et al., 2019), lo que permitiría su conservación durante largos períodos de tiempo evitando las pérdidas debidas la putrefacción (Santos et al., 2010). En este sentido, se trata de una tecnología que puede realizarse con diferentes estrategias, ya sea de modo industrial o a pequeña escala, por lo que se constituye una alternativa accesible a los pequeños productores por su fácil implementación (escasa manipulación y ausencia de mecanización) y bajo coste. Además, esta tecnología aseguraría la disponibilidad de forrajes en épocas de carencia en sistemas de producción de rumiantes (Santos et al., 2010). Por otra parte, la implementación de esta tecnología en pequeños productores favorece la economía circular alargando la vida de los procesos dado que transforma un residuo en una nueva materia prima (Rivas et al., 2019a).

Por lo anterior, la mayoría de los productores, en especial los de pequeña dimensión que cuentan con escasa disponibilidad de base territorial y nulos recursos económicos, no puedan desarrollar su actividad ganadera de forma óptima, lo que se traduce en una baja productividad en la explotación, por cuanto se hace necesario abordar esta actividad bajo un enfoque multidimensional con vistas a mejorar la calidad y disponibilidad de los subproductos agroindustriales disponibles. Así, estas materias primas pueden someterse a distintos procesos físicos, químicos, biológicos o de adición de cultivos microbianos y enzimas fibrolíticas exógenas que permitan incrementar sus valores nutritivos y digestibilidad. En este sentido, la transformación de subproductos de maíz mediante técnicas de ensilado no sólo mejora la digestibilidad, sino que además incrementa la durabilidad de estos recursos para su utilización en épocas de escasez de alimentos, así como también la autosuficiencia de los productores de pequeña escala (Rangel et al., 2017), y contribuye a la mitigación del cambio climático disminuyendo las emisiones de rumiantes (Rivas et al., 2018b).

Todo lo anterior justifica el interés existente en realizar estudios que contribuyan al desarrollo de estrategias de uso racional de recursos endógenos en los sistemas de producción mixtos, por cuanto el objetivo del presente trabajo se centró en analizar el efecto de la inclusión de urea en el ensilado de rastrojo de maíz y melaza sobre la composición química y la cinética de degradación ruminal in situ de la materia seca y la proteína bruta.

\section{MATERIAL Y MÉTODOS}

Esta investigación se ejecutó en el Laboratorio de Ruminología y Metabolismo Nutricional (RUMEN) de la Universidad Técnica Estatal de Quevedo (UTEQ), situado en el Campus Experimental "La María", km 7.0 de la vía Quevedo-Mocache, provincia de Los Ríos, Ecuador, cuya ubicación geográfica es $01^{\circ} 6^{\prime}$ de latitud sur y $79^{\circ} 29^{\prime}$ de longitud oeste y a una altura de 73 msnm.

El estudio se inició con la cosecha y picado (cortes inferiores a 5,0 cm) de rastrojo de maíz (variedad INIAP-H-551), obtenido en una parcela establecida en el Campus Experimental de la Facultad de Ciencias Pecuarias (FCP) de la UTEQ. Se utilizó una picadora de pasto (Trapp ${ }^{\circledR}$ ES 400, Tapp, Jaraguá do Sul, Brasil). La mezcla base del ensilado se preparó con rastrojo de maíz más melaza al $10 \%$, preparando cuatro tipos de tratamientos al adicionar el 0 (T0), 1 (T1), 2 (T2) y 3\% (T3) de urea en cada caso. Se utilizó una balanza MOBBA BS, (Mobba, Barcelona, España).

Tras la homogeneización manual de la mezcla, se elaboraron 128 microsilos de $3 \mathrm{~kg}$ cada uno (4 repeticiones por tratamiento), utilizando fundas de polietileno para empaque al vacío (0,063 mm de grosor), a efectos de garantizar el ambiente anaeróbico requerido durante el proceso de fermentación, según lo establecido por Pereira et al. (2005), Espinoza et al. (2016) y Montenegro et al. (2018). El aire fue extraído por medio de una bomba de vacío y cada bolsa sellada con cinta adhesiva para evitar el ingreso de aire. Posteriormente, se colocaron en un depósito con una temperatura ambiente de $26 \pm 0,6{ }^{\circ} \mathrm{C}$, sin radiación solar directa. Cada 24 horas hasta las 144 horas, se registró la temperatura (Digital 9.2, Gessa, Urkuliz, España) y el pH (pH-metro digital: Crison Basic 20, Crison Instruments, Barcelona-España). La apertura de los silos se realizó cada siete días (7; $14 ; 21 ; 28 ; 35 ; 42 ; 49$ y 56 días). De cada microsilo se extrajo una muestra de 200 gramos, procediendo a su desecación en una estufa (POL-EKO Aparatura) a $65^{\circ} \mathrm{C}$ y molienda a $1 \mathrm{~mm}$ de tamaño de partícula en un molino tipo Thomas-Wiley. A continuación, se determinó el contenido de materia seca (MS), materia orgánica (MO) y proteína bruta (PB), según AOAC (2000), así como la fibra neutro-detergente ((FND) y fibra ácido-detergente (FAD), de acuerdo a los procedimientos de Van Soest et al. (1991).

La degradabilidad in situ se obtuvo mediante el método de Mehrez y Orskov (1977) utilizando cuatro bovinos machos (Brahman) con peso vivo de $450,3 \pm 35.2$ 
$\mathrm{kg}$, provistos de cánulas ruminal permanente (cuatro pulgadas de diámetro interno, Bar Diamond, Parma, Idaho, EEUU), los cuales se alojaron en cubículos individuales, consumieron pasto saboya (Megathyrsus maximus) y King grass (Cenchrus purpureus) durante el experimento, con libre acceso a sales minerales y agua. Las muestras se incubaron por duplicado en cada animal utilizando bolsas $(15 \mathrm{~cm} \times 10 \mathrm{~cm})$ con un tamaño de poro de $45 \mu \mathrm{m}$. Las bolsas se extrajeron a las $0,3,6,12,24,48$ y 72 horas, se lavaron con agua fría y se congelaron a $-30^{\circ} \mathrm{C}$. Estas se descongelaron en una cámara frigorífica a $4^{\circ} \mathrm{C}$, se lavaron con agua fría, se secaron en estufa de aire forzado a $53^{\circ} \mathrm{C}$ hasta peso constante y se pesaron. Los residuos se procesaron por método Kjeldhal (AOAC, 2000) para determinar la degradabilidad de MS y PB.

La desaparición de MS y PB se ajustó a la ecuación $\mathrm{p}=\mathrm{a}+\mathrm{b} \times\left(1-\mathrm{e}^{-\mathrm{ct}}\right)$ (Orskov y Mcdonald, 1979), donde $p$ es la desaparición de MS a tiempo t, a es la fracción soluble por lavado de las bolsas a la hora $0(\%), b$ es la fracción insoluble pero potencialmente degradable (\%), y c es la tasa de degradación de $\mathrm{b}\left(\mathrm{h}^{-1}\right)$. La degradabilidad efectiva (DEMS y la DEPB) se calculó para tres tasas de paso ruminal (k): 0,02, 0,05 y 0,08 $\mathrm{h}^{-1}$, de acuerdo con la ecuación DEMS $=\mathrm{a}+[(\mathrm{b} \times \mathrm{c}) /(\mathrm{c}+\mathrm{k})]$, donde $\mathrm{a}, \mathrm{b}, \mathrm{c}$ y k se han descrito anteriormente.

Todos los análisis estadísticos se hicieron con SAS 9,1 (SAS Institute Inc., Cary, NC, EUA) (SAS Institute Inc. 2004). Los datos se analizaron con el procedimiento GLM y las medias de mínimos cuadrados se compararon con el test de Tukey $(\mathrm{P} \pm<0.05)$. Las respuestas lineales de las variables a la inclusión de niveles de urea se analizaron mediante contrastes polinómicos ortogonales con la opción CONTRAST. Los parámetros de la cinética de degradación se calcularon con el modo de resolución GRG NONLINEAR de la función SOLVER de Microsoft EXCEL ${ }^{\circledR}$.

Para establecer la relación funcional entre la degradabilidad y los niveles de urea, se analizaron las ecuaciones de regresión (lineales, cuadráticas, cúbicas, logarítmicas y Gompertz) y se utilizó el método descendente. Para la selección de la ecuación de mejor ajuste se consideró valor más elevado de $\mathrm{R}^{2}$, alta significación, bajo error estándar de los términos y de estimación, menor cuadrado medio del error, aporte significativo de los términos de la ecuación y bajo coeficiente de indeterminación (1- $\left.\mathrm{R}^{2}\right)$.

\section{RESULTADOS}

La Tabla I muestra la composición química del rastrojo de maíz solo y combinado con diferentes niveles de urea $(0,1,2$ y 3\%) más la adición de melaza, destacando el escaso valor de PB que oscila desde el 3,85 al 5,90\%. El mejor comportamiento para el tiempo de ensilado se observó a los 35 días en comparación al resto de tratamientos $(\mathrm{p}<0,05)$, así la composición química obtenida se detalla en la Tabla II, así como también se expone el resultado del análisis de varianza utilizando el porcentaje de adición de urea como factor de variación. Se evidencia la existencia de diferencias significativas para todas las variables estudiadas excepto MO. Se vislumbró un comportamiento diferenciado entre el ensilado sin adición de melaza respecto al resto de tratamientos que contenían diferentes niveles tanto en MS y PB como FND y FAD, de manera que la adición de urea se relacionó con el incremento de MS y PB y con el descenso de valores de FND y FAD $(p<0,01)$.

Los resultados sobre la cinética de la degradabilidad ruminal In situ de MS se muestran en la Tabla III donde se pudo observar que los valores en las distintas variables van ascendiendo desde mínimos establecidos para T0 hasta máximos reportados para T3. Asimismo, el análisis de varianza reflejó la existencia de diferencias significativas en todas las variables analizadas, según el tratamiento del ensilado, excepto en el caso del factor c. Así, quedan claras las diferencias existentes entre los ensilados con y sin adición, así como, generalmente, entre el nivel de adición de urea al 3\% y el resto.

Tabla I. Composición química (\%) del rastrojo de maíz (Zea mays) con niveles crecientes de urea (Chemical composition of maize stubble (Zea mays) with increasing levels of urea).

\begin{tabular}{cccccc}
\multirow{2}{*}{ Variables } & \multirow{2}{*}{$\begin{array}{c}\text { Rastrojo } \\
\text { de maíz }\end{array}$} & \multicolumn{4}{c}{$\begin{array}{c}\text { Rastrojo de maíz, melaza y niveles cre- } \\
\text { cientes de urea (\%) }\end{array}$} \\
\cline { 3 - 6 } & & 0 & 1 & 2 & 3 \\
\hline MS & 95,81 & 98,39 & 97,99 & 97,48 & 98,77 \\
MO & 93,20 & 89,33 & 90,16 & 90,54 & 90,66 \\
Cenizas & 6,80 & 10,67 & 9,84 & 9,46 & 9,34 \\
PB & 3,85 & 4,90 & 5,67 & 5,66 & 5,90 \\
FND & 72,25 & 64,41 & 62,54 & 60,39 & 64,92 \\
FAD & 46,55 & 40,61 & 40,69 & 38,53 & 40,39 \\
\hline
\end{tabular}

MS: materia seca; MO: materia orgánica; PB: proteína bruta, FND: fibra neutro detergente; FAD: fibra ácido detergente

Tabla II. Valores promedio y análisis de varianza para ensilados de rastrojo de maíz (Zea mays) con niveles crecientes de urea a los 35 días de fermentación (Average values and analysis of variance for maize stubble silages (Zea mays) with increasing levels of urea at 35 days of fermentation).

\begin{tabular}{ccccccc}
\hline \multirow{7}{*}{ Variables } & \multicolumn{6}{c}{ Rastrojo de maíz, melaza y niveles } \\
\cline { 2 - 7 } crecientes de urea (\%)
\end{tabular}

MS: materia seca; MO: materia orgánica; PB: proteína bruta, FND: fibra neutro detergente; FAD: fibra ácido detergente; EEM: error estándar de la media; P: nivel de significación; letras iguales en la misma fila: homogeneidad estadística; letras diferentes en la misma fila: diferencias estadísticas $p<0,01$. 
En la Tabla IV se exponen los resultados sobre la cinética de la degradabilidad ruminal In situ de PB donde se apreció la existencia de homogeneidad estadística entre los distintos tratamientos para las variables factor (a), factor (b), factor (c) y DEPB 8\%, mientras que si evidenciaron diferencias significativas entre tratamientos para el resto de las variables. Así, se conformaron dos agrupamientos para DEPB 2\% y DEPB 5\%, de manera que T0 se separó claramente respecto al grupo com- puesto por T1, T2 y T3, correspondiendo los valores menores a T0.

En el caso de DP, el comportamiento fue similar al de DEPB 2\% y DEPB 5\% con la excepción que T1 compartió grupo de homogeneidad con T0 y con T2-T3.

\section{DISCUSIÓN}

Los residuos de cosecha desempeñan un papel preponderante en los sistemas mixtos agrícolas y pecua-

Tabla III. Valores promedio y análisis de varianza para la cinética de degradabilidad ruminal in in situ de la materia seca del ensilado de rastrojo de maíz (Zea mays) con niveles crecientes de urea a los 35 días de fermentación (Average values and analysis of variance for in situ ruminal degradation kinetics of dry matter from maize stubble silage (Zea mays) with increasing levels of urea at 35 days of fermentation).

\begin{tabular}{|c|c|c|c|c|c|c|}
\hline \multirow{2}{*}{ Parámetro } & \multicolumn{4}{|c|}{ Nivel de urea (\%) } & \multirow{2}{*}{ EEM } & \multirow{2}{*}{$P$} \\
\hline & 0 & 1 & 2 & 3 & & \\
\hline $\mathrm{a}, \% \mathrm{MS}$ & $26,58^{b}$ & $32,00^{a}$ & $30,39^{a}$ & $30,97^{a}$ & 1,23 & $<0,044$ \\
\hline $\mathrm{b}, \% \mathrm{MS}$ & $27,16^{b}$ & $27,87^{\mathrm{b}}$ & $31,71^{\mathrm{ab}}$ & $33,61^{a}$ & 1,55 & $<0,036$ \\
\hline $\mathrm{c}, \mathrm{h}^{-1}$ & $0,05^{\mathrm{a}}$ & $0,03^{a}$ & $0,04^{a}$ & $0,04^{a}$ & 0,01 & $>0,426$ \\
\hline $\mathrm{DP}, \% \mathrm{MS}$ & $53,74^{c}$ & $59,87^{b}$ & $62,10^{\mathrm{ab}}$ & $64,58^{a}$ & 0,95 & $<0,000$ \\
\hline DEMS $2 \% \mathrm{~h}^{-1}$ & $44,94^{d}$ & $48,12^{c}$ & $5074^{b}$ & $53,54^{a}$ & 0,65 & $<0,000$ \\
\hline DEMS 5\% $\mathrm{h}^{-1}$ & $39,10^{c}$ & $41,88^{b}$ & $43,65^{b}$ & $46,15^{a}$ & 0,81 & $<0,000$ \\
\hline DEMS $8 \% \mathrm{~h}^{-1}$ & $36,13^{c}$ & $39,12^{b}$ & $40,22^{\mathrm{ab}}$ & $42,41^{a}$ & 0,88 & $<0,002$ \\
\hline
\end{tabular}

EEM: Error Estándar de la Media; MS: Materia Seca; a: fracción soluble; b: fracción potencialmente degradable; c: tasa de degradación de b; DP: Degradabilidad Potencial (a+b); DEMS: Degradabilidad Efectiva de la Materia Seca a tasas de paso ruminal del 2,5 y $8 \%$ h¹. $a, b, c$ Las medias de mínimos cuadrados sin un superíndice común son significativamente diferentes $(P<0.05)$.

Tabla IV. Valores promedio y análisis de varianza para la cinética de degradabilidad ruminal in vivo de la proteína bruta del ensilado de rastrojo de maíz (Zea mays) con niveles crecientes de urea a los 35 días de fermentación (Average values and analysis of variance for in vivo ruminal degradation kinetics of crude protein from maize stubble silage (Zea mays) with increasing levels of urea at 35 days of fermentation).

\begin{tabular}{|c|c|c|c|c|c|c|}
\hline \multirow{2}{*}{ Parámetro } & \multicolumn{4}{|c|}{ Nivel de urea (\%) } & \multirow{2}{*}{ EEM } & \multirow{2}{*}{$P$} \\
\hline & 0 & 1 & 2 & 3 & & \\
\hline$a, \%$ PB & $13,84^{\mathrm{a}}$ & $18,81^{a}$ & $19,41^{a}$ & $20,79^{a}$ & 3,24 & $>0,482$ \\
\hline$b, \%$ PB & $23,94^{\mathrm{a}}$ & $30,08^{a}$ & $33,52^{\mathrm{a}}$ & $33,77^{a}$ & 5,74 & $>0,606$ \\
\hline $\mathrm{c}, \mathrm{h}^{-1}$ & $0,15^{\mathrm{a}}$ & $0,08^{a}$ & $0,07^{a}$ & $0,06^{a}$ & 0,06 & $>0,679$ \\
\hline $\mathrm{DP}, \% \mathrm{~PB}$ & $37,78^{\mathrm{b}}$ & $48,89^{\mathrm{ab}}$ & $52,92^{\mathrm{a}}$ & $54,56^{\mathrm{a}}$ & 3,68 & $<0,029$ \\
\hline DEP $2 \% \mathrm{~h}^{-1}$ & $30,03^{b}$ & $42,14^{a}$ & $41,97^{\mathrm{a}}$ & $44,34^{\mathrm{a}}$ & 1,93 & $<0,000$ \\
\hline DEP $5 \% \mathrm{~h}^{-1}$ & $25,48^{b}$ & $36,38^{a}$ & $35,34^{a}$ & $37,39^{a}$ & 2,65 & $<0,028$ \\
\hline DEP $8 \% \mathrm{~h}^{-1}$ & $23,23^{b}$ & $32,95^{\mathrm{ab}}$ & $31,94^{\text {ab }}$ & $33,72^{\mathrm{a}}$ & 3,03 & $<0,101$ \\
\hline
\end{tabular}

EEM: Error Estándar de la Media; PB: Proteína Bruta; a: fracción soluble; b: fracción potencialmente degradable; c: tasa de degradación de b; DP: Degradabilidad Potencial ( $\mathrm{a}+\mathrm{b})$; DEPB: Degradabilidad Efectiva de la Proteína Bruta a tasas de paso ruminal del 2,5 y $8 \%$ h ${ }^{-1}$. a,b,c Las medias de mínimos cuadrados sin un superíndice común son significativamente diferentes $(P<0.05)$.

rios en el mundo, según Camacho et al., (2014), si bien su uso como alimentación animal se viene compartiendo con las necesidades de cobertura del suelo de la agricultura de conservación en las últimas décadas, siendo muy escaso su empleo como biomasa para la fabricación de bioetanol (García-Stepien et al. 2016. Desde la perspectiva pecuaria, han sido muchos los estudios orientados hacia la evaluación de los subproductos agroindustriales en términos nutricionales, de digestibilidad, eficiencia y desempeño (García-Martí- nez et al., 2009; Espinoza, et al., 2016; López-Inzunza, et al. 2017; y Montenegro et al., 2018, entre otros), así como su utilización bajo criterios de sostenibilidad (Erenstein et al., 2011 y Hellin et al., 2013). La utilización del rastrojo de maíz está muy extendida en todo el continente americano dado que se trata del residuo agrícola mayoritario, ya sea por la amplia extensión de su cultivo como en el caso de Ecuador (INEC, 2018), como por su rendimiento (Muñoz, 2011). 
Los resultados obtenidos sobre la composición química del rastrojo de maíz fueron coincidentes con los reportados en México por Fuentes et al. (2001) y López Aguirre et al. (2015), a excepción del valor de $\mathrm{PB}$, el cual se mostró en torno a $1 \%$ inferior, así como sensiblemente diferentes a los expuestos por SánchezAcosta et al. (2012) también en México, de manera que, en nuestro estudio, se observaron mayores valores en MS, MO, FND y FAD y claramente inferiores en PB y cenizas. Esta situación podría explicarse atendiendo a la alta variabilidad existente entre las múltiples variedades de maíz cultivadas, tal y como se refleja en el trabajo de Muñoz-Tlahuiz et al. (2013) al valorar 144 variedades de esta especie. La composición química de la mezcla inicial de ensilado presentó valores inferiores para todas las variables en los distintos tratamientos de urea a los expuestos por Fuentes et al. (2001), si bien en este caso se incorporó un $4 \%$ de urea a la mezcla del ensilado y no contó con adición de melaza.

Múltiples estudios han constatado la bondad de la técnica de ensilaje con relación a la modificación de la composición química y mejora de la digestibilidad, como Castillo et al. (2009), Nkosi et al. (2009) y Thomas et al. (2013), entre otros, cuestión que también pudo corroborarse en el presente trabajo, así como se evidenció T2 como el tratamiento de elección en el caso del rastrojo de maíz, al contar con el nivel más alto de PB y menor proporción de FAD. En el caso de Ecuador, ese mismo comportamiento ha sido puesto de manifiesto al estudiar el ensilado de distintos subproductos agroindustriales como residuos de maracuyá (Espinoza et al., 2017), así como también residuos de piña y plátano (Montenegro et al., 2018).

El efecto significativo de la inclusión de urea en el ensilado sugiere una mejor utilización de MS a nivel general, y de PB a nivel particular, atendiendo a los parámetros de cinética de degradabilidad ruminal in situ, así como los valores de degradabilidad potencial y efectiva. Esta situación puede deberse a la mayor disponibilidad de solubles neutro detergentes (SND = 100 - FND) por parte de los microorganismos ruminales. Asimismo, el aumento de la DEMS y DEPB se relaciona con una mejora del valor energético del ensilado con la inclusión del 3\% de urea, la cual podría estimarse en 0,21 megajulios (MJ)/ $\mathrm{kg}$ MS para rumiantes.

Aunque existen referencias de la inclusión de un $4 \%$ de urea en ensilado de rastrojo de maíz para la alimentación de ovino (Sánchez et al., 2012), un factor limitante en nuestro estudio es el desconocimiento del efecto de niveles superiores de dicho aditivo sobre la degradabilidad de la mezcla, así como respecto al límite de tolerancia de los animales, especialmente en el caso del bovino. En este sentido, el trabajo experimental se realizó utilizando bovinos fistulados de raza Brahman en el laboratorio acreditado de la Universidad Técnica Estatal de Quevedo (UTEQ) de Ecuador, si bien la población bovina preponderante en nuestra área de estudio es el ganado criollo de doble propósito, siendo recomendable ampliar estudios de degradabilidad específicos en estos animales al constituir la base productiva de la región como estrategia de desarrollo rural en el futuro.
Los resultados obtenidos contribuyen al conocimiento de la utilización del ensilado de rastrojo de maíz en las condiciones de Ecuador, pudiendo recomendarse su uso en los sistemas de producción de rumiantes durante los períodos de escasez de alimento. Esta modalidad de conservación de forrajes que, minimiza las pérdidas de materia prima tanto en el proceso de transformación como en la fase de almacenamiento, así como permite su elaboración en la explotación en aquellos intervalos de tiempo sin actividad agrícola (Sánchez et al., 2019), puede emplearse, al menos, como ración de mantenimiento de los animales, coincidiendo con Guzmán et al. (2019). Igualmente, también podría ser viable su utilización como un ingrediente más de la dieta en sintonía con el estudio de SánchezAcosta et al. 2012). En cualquier caso, los valores nutricionales superan a los del rastrojo de maíz cuando los productores optan por su uso directo (Arellano-Vicente et al. 2016).

El ensilado de rastrojo de maíz con adición de melaza y urea constituye una adopción tecnológica de fácil implementación y bajo coste, la cual puede convertirse en el eje vertebrador de los sistemas mixtos y de producción integrada en Ecuador como herramienta dinamizadora de desarrollo rural bajo criterios de sostenibilidad, de mitigación y adaptación al cambio climático (Galindo-Barboza et al., 2020) y economía circular (Ward et al., 2016).

\section{CONCLUSIONES}

La utilización de rastrojo de maíz y su combinación con melaza y urea mediante técnicas de ensilado mejora las características nutritivas iniciales del ingrediente base, pudiéndose erigir como una alternativa de suplementación animal eficiente y aceptable ambientalmente por parte de los productores de pequeña escala en su área de producción. Nuestros resultados evidenciaron la existencia de un marcado efecto de la inclusión de melaza y urea en la mezcla de ensilado sobre la degradabilidad de la materia seca y proteína bruta, así como un adecuado comportamiento de los parámetros de la cinética de degradabilidad. Asimismo, estos datos sugieren que la mejor elección sería el tratamiento con un nivel de inclusión del 3\% de urea.

\section{AGRADECIMIENTOS}

Este trabajo ha sido realizado en el seno del proyecto de investigación denominado "Composición química, ensilabilidad, estabilidad aeróbica de dos subproductos agrícolas para la alimentación de rumiantes en el Litoral ecuatoriano", desarrollado por la Universidad Técnica Estatal de Quevedo (UTEQ) en Ecuador y la Universidad de Córdoba en España, el cual fue financiado a través del Fondo Competitivo de Investigación de Ciencia y Tecnología (FOCICYT).

\section{BIBLIOGRAFÍA}

Association of Official Analytical Chemists 2000, 'Official Methods of Analysis', 21 st edn, AOAC, Washington DC.

Arellano-Vicente, I, Pinto-Ruíz, R, Guevara-Hernández, F, Reyes-Muro, L, Hernández-Sánchez, D, \& Ley de Coss, A 2016, 'Caracterización 
del uso directo de rastrojo de maíz (Zea mays L.) por bovinos', Revista Mexicana de Ciencias Agrícolas, vol. 7, n 5, pp. 1117-1129.

Camacho, TC, Espinosa, JA, Borja, M, Reyes, L, Guevara, F 2014, 'Antecedentes', in Reyes, ML, Camacho, TC, \& Guevara, F, (eds.), Rastrojos manejo, uso y mercado en el Centro y Sur de México. INIFAP. México.

Cándido, M, Neiva, J, Rodríguez, N, \& Ferreira, A 2007, 'Características fermentativas e composição química de silagens de capim-elefante contendo subproduto desidratado do maracujá', Revista Brasileira de Zootecnia, vol. 36, n 5, pp. 1489-1494.

Caraballo, A, Betancourt, M, \& Florio, J 2007, 'Efecto de la melaza, estado fisiológico del pasto y tamaño del material cosechado sobre el ensilado de pasto guinea (Panicum maximum, Jacq.) ', Ciencia, vol. 15, n 1, pp. 35-46.

Castillo, JM, Rojas, BA, \& Wingching, JR 2009, 'Valor nutricional del ensilaje de maíz cultivado en asociación con vigna (Vigna radiata)', Agronomía Costarricense, vol. 33, n 1, pp. 133-146.

Erenstein, O, Samaddar, A, Teufel, N, \& Blummel, M 2011 , 'The paradox of limited maize stover use in India's smallholder crop-livestock systems', Experimental Agriculture, vol. 47, $\mathrm{n}^{\circ}$ 4, pp. 677-704

Espinoza, I, Pérez, C, Montenegro, L, Sánchez, A, García, A, \& Martínez, A 2016, 'Composición química y cinética de degradación ruminal in vitro del ensilado de pasto saboya (Megathyrsus maximus) con niveles crecientes de inclusión de residuos de maracuyá (Passiflora edilus Sims.)', Revista Científica FVC-LUZ, vol. XXVI, n 6, pp. 402-407.

Espinoza, I, Montenegro, L, Rivas, J, Romero, M, García, \& Martínez, A 2017, 'Características microbianas, estabilidad aeróbica y cinética de degradación ruminal del ensilado de pasto saboya (Megathyrsus maximus) con nivel crecientes de cáscara de maracuyá (Passiflora edulis)', Revista Científica FVC-LUZ, vol. XXVII, nº 4, pp. 241-248.

Fuentes, J, Magaña, C, Suárez, L, Peña, R, Rodríguez, S, \& Ortiz de la Rosa, B 2001, 'Análisis químico y digestibilidad "in Vitro" de rastrojo de maíz (Zea mays L.)', Agronomía Mesoamericana, vol. 2, n 12, pp. 189-192.

Galindo-Barboza, AJ, Domínguez-Araujo, G, Arteaga-Garibay, RI, \& Sálazar-Gutiérrez, G 2020, 'Mitigación y adaptación al cambio climático mediante la implementación de modelos integrados para el manejoy aprovechamiento de los residuos pecuarios. Revisión', Revista Mexicana de Ciencias Pecuarias, vol. 11, supl. 2, pp. 107-125

García, A, Rivas, J, Rangel, J, Espinosa, JA, Barba, C, \& De PablosHeredero, C 2016, 'A methodological approach to evaluate livestock innovations on small-scale farms in developing countries', Future Internet, vol. 8, pp. 1-17.

Garcia-Martinez, A, Albarran-Portillo, B, Castelan-Ortega, OA, EspinozaOrtega, A, \& Arriaga-Jordan, C 2009, 'Urea treated maize straw for small-scale dairy systemsin the highlands of Central Mexico', Tropical Animal Health and Production, vol. 41, n 7, pp. 1487-1494.

García-Stepien LE, Borlandelli, MS, Roldán, DO, \& Ibánez, YM 2016, 'Rastrojo de maíz: producción teórica de etanol lignocelulósico en relación a la fecha de siembra', In: IIPAAS (ed), Cuarto Congreso Internacional Científico y Tecnológico de la provincia de Buenos Aires, Buenos Aires, Argentina, Consultado 5 abril 2020, https:// digital.cic.gba.gob.ar/bitstream/handle/1 1746/6854/14-IIPAS. pdf? sequence $=1$ \&isAllowed $=y$

Guzmán, O, Lemus-Flores, C, Bugarín, J, Bonillas, J, González-Vizcarra, V, \& Ly J 2019, 'In situ ruminal degradability of silage of mango wastes (Mangifera indica L.) and maize stubbles', Cuban Journal of Agricultural Science, vol. 53, $n^{\circ} 2$, pp. 139-148.

Hellin, J, Erenstein, O, Beuchelta, T, Camacho, C, \& Flores, D 2013, 'Maize stover use and sustainable crop production in mixedcrop-livestock systems in Mexico', Fields Crops Research, vol. 153, pp. 12-21. Instituto Nacional de Estadística y Censos 2018, ‘Encuesta de Superficie y Producción Agropecuaria Continua (ESPAC)' Quito, Ecuador.

López-Aguirre, D, Vázquez-Armijo, JF, Salem, AZM, Hernández-Meléndez, J, Rojo, R, \& Limas, AG 2015, 'Degradabilidad de la materia seca y las fracciones de fibra de forrajes de baja calidad tratados con enzimas exógenas', in AIDA (ed.), Proceedings XVI Jornadas sobre Producción Animal, pp. 197-199

López-Inzunza, HJ, Chongo-García, BB, La O-León, O, Guerra-Liera, JE, Luna-López, M, Castro-Camacho, SJ, \& López-Juárez, LA 2017, 'Digestiblidad in situ de rastrojo de maíz tratado con enzimas fibrolíticas', Revista Ciencia y Agricultura, vol. 14, n 1, pp. 31-37.

Mehrez, AZ, \& Ørskov, E 1977, ‘A study of artificial fiber bag technique for determining the digestibility of feed in the rumen', Journal of Agricultural Science, vol. 88, pp. 645-650.

Meneses, J, Corrales, C, \& Valencia, M 2007, 'Síntesis y caracterización de un polímero biodegradable a partir del almidón de yuca', Revista Escuela Ingeniería Antioquia, vol. 8, pp. 57-67.

Montenegro, L, Espinoza, I, Sánchez, A, Barba, C, García, A, Requena, F, \&Martínez, A 2018, 'Composición química y cinética de degradación ruminal in vitro del ensilado de pasto saboya (Megathyrsus maximus) con inclusión de residuos de frutas tropicales', Revista Científica FVCLUZ, vol. XXVIII, n 4 , pp. 306-312.

Muñoz F 2011, 'Producción, valor nutricional y aprovechamiento del rastrojo de maíces nativos en la región de Libres-Serdán, Puebla, México', Tesis de maestría, Colegio de Postgraduados de México, consultado 5 abril 2020, http://www.biblio.colpos.mx:8080/ispui/ handle/10521/602.

Muñoz-Tlahuiz, F, Guerrero-Rodríguez, JD, López, JA, Gil-Muñoz, A, López-Sánchez, H, Ortíz-Torres, E, Hernández-Guzmán, JA, TaboadaGaytán, O, Vargas-López, S, \& Valadez-Ramírez, M2013, 'Producción de rastrojo y grano de variedades locales de maíz en condiciones de temporal en los valles altos de Libres Serdán, Puebla, México', Revista Mexicana de Ciencias Pecuarias, vol. 4, n 4, pp. 515-530. Nkosi, BD, Meeske, R, Palic, D, Langa, T, Leeuw, KJ, \& Groenewald, IB 2009, 'Effects of ensiling whole crop maize with bacterial inoculants on the fermentation, aerobic stability, and growth performance of lambs', Animal Feed Science Technology, vol. 154, n 3-4, pp. 193-203

Ørskov, E, \& McDonald, J 1979, 'The estimation of protein degradability in the rumen from incubation measurements weighted according to rate of passage', Journal of Agricultural Science, vol. 92, pp. 499-504.

Pardo, G, Martín-García, I, Arco, A, Yañez-Ruiz, D, Moral, R, \& Del Prado, A 2016. 'Greenhouse-gas mitigation potential of agro-industrial by-products in the diet of dairy goats in Spain: a life-cycle perspective', Animal Production Science, vol. 56, n 3, pp. 646-654.

Pereira, L, Gonçalves, L, Tomich, T, Borges, I, \& Rodríguez, N 2005. 'Silos experimentais para avaliação da silagem de três genótipos de girasol (Helianthus annuus L.)', Arquivo Brasileiro de Medicina Veterinária e Zootecnia, vol. 57, n 5, pp. 690-696.

Rangel, J, Espinosa, JA, De Pablos-Heredero, C, Rivas, J, Perea, J, Angón, E, \& García, A 2017, 'Is the increase of scale in the tropics a pathway to smallholders? Dimension and ecological zone effect on the mixed crop-livestock farms', Spanish Journal of Agricultural Research, vol. $15, \mathrm{n}^{\circ} 2, \mathrm{pp} 1-10$

Rangel, J, Perea, J, De Pablos-Heredero, C, Espinoza, JA, Toro-Mújica, P, Fijoó, M, Barba, C, \& García, A 2020, 'Structural and Technological Characterization of Tropical Smallholder Farms of Dual-Purpose Cattle in Mexico', Animals, vol. 86, n 86, pp. 1-13

Rivas, J, Perea, J, De Pablos-Heredero, C, Angón, E, Barba, C, García, A 2019a, 'Canonical correlation of technological innovation and performance in sheep's dairy farms: Selection of a set of indicators', Agricultural Systems, vol. 176, pp. 102665

Rivas, J, Perea, J, De Pablos-Heredero, C, Morantes, M, Angón, E, Barba, C, García, A 2019b, 'Role of technological innovation in livestock breeding programmes: a case of cereal-sheep system', Italian Journal of Animal Science, vol. 18, $\mathrm{n}^{\circ} 1$, pp. 1049-1057

Sánchez-Acosta, E, Ortega-Cerrilla, ME, Mendoza-Martínez, G, Montañez-Valdez, OD, \& Buntix-Dios, SE 2012, 'Rastrojo de maíz tratado con urea y metionina protegida en dietas para ovinos en crecimiento', Interciencia, vol. 37, nº 5, pp. 395-399.

Sánchez-Laiño, A, Torres-Navarrete, E, Espinoza-Guerra, I, MontenegroVivas, L, Barba-Capote, C, \& García-Martínez, A 2019, 'Valoración nutricional in situ de dietas con harina de maracuyá (Passiflora edu- 
lis) en sustitución del maíz (Zea mays)', Revista de Investigaciones Veterinarias del Perú, vol. 30, n 1, pp 149-157

Santos, MVF, Gómez-Castro, AG, Perea, JM, García, A, Guim, A \& Pérez-Hernández, M 2010. 'Factores que afetam o valor nutritivo da silagens de forrageiras tropicais', Archivos de Zootecnia, vol. 59 (R), pp. 25-43.

Thomas, R, Nkosi, BD, Umesiobi, DO, Meeske, R, Kanengoni, AT, \& Langa, T 2013, 'Evaluation of potato hash two silage inoculants bacteria and their effects on the growth of fattening pigs. Sudafricana', Journal of Animal Science, vol. 40, n 5, pp. 488-490.

Torres, G, Arbaiza, T, Carcelén, F, \& Lucas, O 2009, 'Comparación de las técnicas in situ, in vitro y enzimática (celulasa) para estimar la digestibilidad de forrajes en ovinos', Revista de Investigaciones Veterinarias del Perú, vol. 20, n 1, pp 5-9
Torres, Y, García, A, Rivas, J, Perea, J, Angón, E, \& De-Pablos-Heredero, C 2015, 'Socioeconomic and productive characterization of dual-purpose farms oriented to milk production in a tropical region of Ecuador. The case of the province of Manabi', Revista Científica FVC-LUZ, vol. XXV, $n^{\circ} 25$, pp. 330-337.

Van Soest, PJ, Robertson, JB, \& Lewis, BA 1991, Methods for dietary fiber, neutral fiber and no starch polysaccharides in relation to nutrition', Journal Dairy Science, vol.74, $\mathrm{n}^{\circ} 10$, pp. 3583-3597.

Ward, SM, Holden, NM, White, EP, \& Oldfield, TL 2016, 'The "Circular Economy" applied to the agriculture (livestock production) sector - Discussion paper', in Workshop on the Sustainability of the EU's Livestock Production Systems Hosted, DG Agriculture and Rural Development, European Commission, Brussels, Belgium 